\title{
AUSENTISMO, INDECISIÓN Y CAMPAÑAS DIGITALES EN LA CARRERA HA- CIA LAS ALCALDÍAS 2019 EN ECUADOR
}

\author{
Authentication, Indecision and Digital Campaigns in the Race to the Mayors 2019 in Ecuador
}

\author{
FÉLIX WILMER PAGUAY CHÁVEZ \\ Universidad Politécnica Estatal del Carchi \\ felixpaguay@hotmail.es; fwpaguay@pucesi.edu.ec
}

\author{
CRISTINA ALEXANDRA VÁSQUEZ CADENA \\ Pontificia Universidad Católica del Ecuador, Sede Ibarra \\ cavasquez@pucesi.edu.ec; cvasquezcadena@gmail.com

\section{CARMELO MÁRQUEZ-DOMÍNGUEZ \\ Pontificia Universidad Católica del Ecuador, Sede Ibarra \\ camarquez@pucesi.edu.ec; carmelomarquez@gmail.com}

Resumen: El presente trabajo de investigación se elaboró bajo el enfoque cuantitativo, basado en el tipo de investigación correlacional, descriptiva y no experimental; sobre la base de datos de las elecciones seccionales 2019 de los 221 cantones del Ecuador, se propuso determinar la relación entre los votos válidos, el ausentismo, los votos nulos, votos blancos y el número de candidatos a las alcaldías, así como también, se buscó establecer el nivel de eficiencia de la estrategia de comunicación política mediante la tasa de conversión de seguidores de las plataformas Facebook y Twitter a votos válidos. Entre los principales resultados se destaca por un lado la correlación significativa entre las variables de estudio, por ejemplo, los votos blancos y nulos presentaron una relación positiva y significativa con el ausentismo $(0.850$ y 0.867 , respectivamente), mientras que por otro, las tasas de conversión sugieren un desempeño eficiente de la estrategia de comunicación en las plataformas sociales; sin embargo, es un error generalizar estos resultados como un factor determinante en las elecciones seccionales de 2019, porque los estrategas políticos en Ecuador, recurren generalmente a las tácticas de comunicación política a través de medios de comunicación tradicionales.

Palabras clave: Facebook; Twitter; Elecciones; Votos válidos; Ecuador; Comunicación política; tasa de conversión; Medios; CNE.

\begin{abstract}
This research work was developed under the quantitative approach, based on the type of correlational, descriptive and non-experimental research; Based on data from the 2019 sectional elections of the 221 cantons of Ecuador, it was proposed to determine the relationship between valid votes, absenteeism, null votes, white votes and the number of mayoral candidates, as well as He sought to establish the level of efficiency of the political communication strategy through the conversion rate of followers of the Facebook and Twitter platforms to valid votes. Among the main results, the significant correlation between the study variables stands out, for example, white and null votes presented a positive and significant relationship with absenteeism ( 0.850 and 0.867 , respectively), while on the other, the rates Conversion suggests an efficient performance of the communication strategy on social platforms; However, it is a mistake to generalize these results as a determining factor in the sectional elections of 2019, because political strategists in Ecuador generally resort to political communication tactics through traditional media.
\end{abstract}

Keywords: Facebook; Twitter; Elections; Valid votes; Ecuador; Political communication; Conversion rate; Media; $C N E$. 


\section{INTRODUCCIÓN}

Las investigaciones relacionadas con el comportamiento del votante, adoptan dos enfoques claramente definidos: el primero abarca estudios que se concentran en explicar por qué los electores votan por determinado candidato que representa a un partido político; $y$, el segundo enfoque contempla estudios que abordan a la abstención, los votos blancos y nulos como alternativas que representan posiciones diferentes frente a las propuestas de los candidatos (Clarke, et al, 2011). El presente estudio optó por el segundo enfoque, el cual ofrece algunas bases conceptuales respecto a los términos abstención, votos blancos y nulos que permita comprender los resultados que dejaron las elecciones de alcaldes en Ecuador.

Las pasadas elecciones seccionales, celebradas el 24 de marzo del 2019, buscaban renovar a las autoridades, parroquiales, cantonales y provinciales, y los resultados presentaron ciertas particularidades; las más destacadas fueron el número de candidatos, además, la reestructuración del mapa político de Ecuador en donde la fuerza más importante es el Partido Social Cristiano; mientras CREO y Alianza País pierden posiciones estelares.

Tabla 1. Fuerzas Políticas según el voto acumulado en prefecturas y alcaldías.

\begin{tabular}{|c|c|c|c|}
\hline & Prefecturas & Alcaldías & Voto Acumulado \\
\hline $\begin{array}{l}\text { Partido Social } \\
\text { Cristiano }\end{array}$ & $1^{\prime} 716.457$ & $1 ' 934.882$ & 3'651.339 \\
\hline $\begin{array}{l}\text { Movimiento } \\
\text { Democracia Sí }\end{array}$ & 923.034 & 1'356.560 & $2 ’ 279.594$ \\
\hline $\begin{array}{l}\text { Movimiento } \\
\text { CREO }\end{array}$ & 1'109.894 & 909.546 & $2^{\prime} 019.440$ \\
\hline $\begin{array}{l}\text { Movimiento F. } \\
\text { Compromiso } \\
\text { Social }\end{array}$ & 977.059 & 519.048 & $1 ' 496.107$ \\
\hline $\begin{array}{l}\text { Movimiento } \\
\text { Alianza PAIS }\end{array}$ & 606.743 & 695.516 & $1 ’ 302.259$ \\
\hline $\begin{array}{l}\text { Centro } \\
\text { Democrático }\end{array}$ & 299.443 & 940.633 & $1 ’ 240.076$ \\
\hline $\begin{array}{l}\text { Izquierda } \\
\text { Democrática }\end{array}$ & 636.806 & 582.167 & $1 ’ 218.973$ \\
\hline
\end{tabular}

Fuente: Resultados CNE/ El Universo

Dichas particularidades son notables por los fallidos pronósticos de las encuestas (Giuliani, 2019) con respecto a los resultados de las alcaldías, aunque también se evidenció un elevado porcentaje de ausentismo e indecisión de los electores. La mayoría de los candidatos ganadores de las alcaldías, en promedio, triunfaron con un $35 \%$ de los votos válidos.

El propósito del artículo es determinar si existe alguna relación entre los resultados electorales obtenidos, el ausentismo, los votos blancos, los votos nulos y el número de 
candidatos a las alcaldías de los 221 cantones del Ecuador, y además, se busca establecer el nivel de eficiencia de la estrategia de comunicación política, mediante el análisis de la tasa de conversión de seguidores a votos válidos de las plataformas Facebook y Twitter.

En este sentido, el trabajo se encuentra estructurado en tres partes, la primera es un marco conceptual que describe a detalle las variables de estudio, la segunda parte expone la metodología, el cómo se procesaron las variables; y la tercera muestra los principales resultados de investigación.

\section{EL COMPORTAMIENTO ELECTORAL EN AMÉRICA LATINA}

Los últimos acontecimientos electorales en América Latina presentan ciertos cambios en el comportamiento electoral, por ejemplo, en las presidenciales peruanas de 2016, hubieron tres dimensiones y actitudes políticas en la decisión electoral de los ciudadanos: las orientaciones izquierda-derecha; las actitudes hacia la intervención del Estado en la economía ("estatismo económico"); las actitudes hacia el ejercicio discrecional del poder político en el gobierno ("mano dura"); como producto de las diferencias programáticas dentro de un sistema de partidos poco institucionalizado con una alta volatilidad electoral y niveles de ausentismo considerables (Sulmont, 2018, p.429).

Por otra parte, una estabilidad electoral tampoco es garantía de una reducción en el ausentismo y el abstencionismo; en Chile, durante el período 1993-2009 existió una importante caída de los votantes fijos y un cuadro de movilidad en el electorado (Avendaño y Sandoval, 2016, p.175), de hecho la estabilidad en el comportamiento electoral no puede generalizarse, las diferentes contiendas electorales son únicas y la probabilidad de encontrar igualdades es muy baja.

Durante la última década, en América Latina los escenarios electorales se caracterizaron por la presencia de ganadores consecutivos y traslados de los apoyos que generalmente estuvieron asociados con variaciones en los niveles de confianza en los partidos políticos. (Barreda y Ruiz, 2019, p.247).

En Ecuador los cambios en las contiendas y en el comportamiento electoral, pueden entenderse si se compara el antes y el durante (donde los niveles del ausentismo se redujeron) de Rafael Correa (El Universo, 2019). El presente trabajo presenta una perspectiva de las contiendas electorales post Correato.

Considerando lo anterior, en el siguiente apartado se describen los elementos que caracterizaron a los comicios celebrados en marzo de 2019, en los cuales se eligieron a las autoridades seccionales y a los nuevos vocales del Consejo de Participación Ciudadana y Control Social; sin embargo, se prioriza en el análisis, el proceso de la elección de los alcaldes de los 221 cantones.

\section{LA PARTICULARIDAD DE LAS ELECCIONES DE MARZO DEL 2019}

En las elecciones seccionales de 2019, se presentaron algunos datos curiosos, primero la fragmentación del movimiento oficialista (correistas y morenistas) debilitaron la principal fuerza política de los últimos diez años; el segundo dato interesante se refiere a la poca actividad de campaña del presidente Moreno, mientras los líderes contrincantes Jaime Nebot del 
Partido Social Cristiano y Guillermo Lasso del movimiento CREO, desarrollaron un trabajo minucioso recorriendo todo el país; finalmente, el tercer dato importante es la cantidad de candidatos participantes a las alcaldías, una muestra de ello es que solamente considerando las cabeceras cantonales de las 24 provincias del Ecuador, el promedio de candidatos a las alcaldías asciende a 11 .

\section{LA ABSTENCIÓN ELECTORAL}

La abstención electoral es "la no participación por parte de quienes tienen derecho a hacerlo, (...) también actúa como un regulador del funcionamiento del sistema democrático" (Milanese; Abadía; Manfredi, 2016, p.79). Domper-Lasús (2018); Coutigno (2018); Southern; Lee (2018); y Müller; Jankowski (2018), por ejemplo, explican que la abstención puede asociarse con la oposición al régimen, cuando el contexto no es democrático; sin embargo, es un fenómeno presente tanto en democracias consolidadas como en desarrollo. (Vasquez-Feria, 2019, p.3)

Partiendo de la premisa de que cada proceso electoral es único, en las elecciones de marzo 2019, la cantidad de candidatos a las alcaldías constituye una característica única respecto de otros procesos electorales desarrollados en Ecuador. Algunas lecturas referidas a las elecciones de marzo describen esta participación de candidatos como señal de buena salud del sistema democrático del país, esta afirmación puede corroborase, si se considera que los márgenes de la abstención se reducen paulatinamente con respecto a 2002.

Figura 1. El ausentismo durante el período 2002-2017.

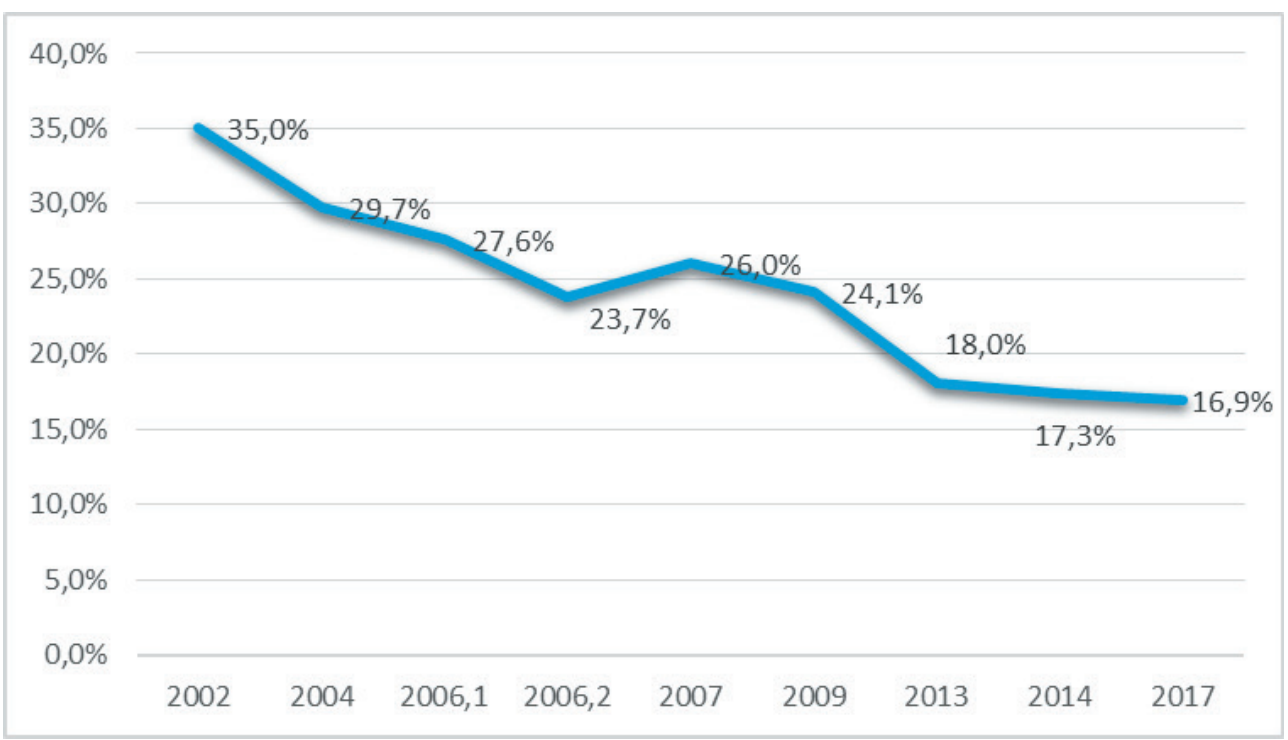

Fuente: Bases de datos de CNE 
La abstención se presenta como un fenómeno multifactorial que no obedece a las leyes de la causalidad (Vasquez-Feria, 2019; Ávila-Eggleton; Gutiérrez-Sánchez, 2017), aunque puede descomponerse en dos actitudes: a) actitud de castigo, algunos electores no votan para mostrar descontento; y b) la desafección política, los electores no votan porque no les interesa la política. (Cazorla; Rivera; Jaráiz, 2017; Ochoa-Villa, 2015).

\section{EL VOTO EN BLANCO Y EL VOTO NULO}

Votar en blanco o nulo responde a una actitud de castigo; Lozano-García y Rodríguez-Casallas (2015) denominan a estas decisiones como abstencionismo cívico, "[...] ocurre cuando el elector participa en la votación, pero deposita en la urna una boleta en blanco o la anula y no contribuye directamente al éxito de la elección [...]" (p.140). Este tipo de comportamientos, también obedecen a la desafección política, que ocurre en cualquier sistema electoral, incluso donde votar es obligatorio, otra posible razón está relacionada con aspectos técnicos, "referidos al diseño de la papeleta, cuando existe gran cantidad de candidatos". (Lozano-García; Rodríguez-Casallas, 2015, p.144).

El comportamiento del voto en blanco y el voto nulo es irregular (ver figura 2); sin embargo, en los procesos electorales del 2006 y 2017, ambos en segunda vuelta, la abstención cívica se reduce, probablemente se deba a la facilidad para escoger de entre uno de los dos candidatos a presidente. Mientras los valores elevados en 2006 primera vuelta y en 2009 pueden tener relación con el aspecto técnico porque en ambos casos coincidieron las elecciones generales y seccionales, donde se triplican las dignidades en disputa.

Figura 2. Comportamiento del voto en blanco y el voto nulo.

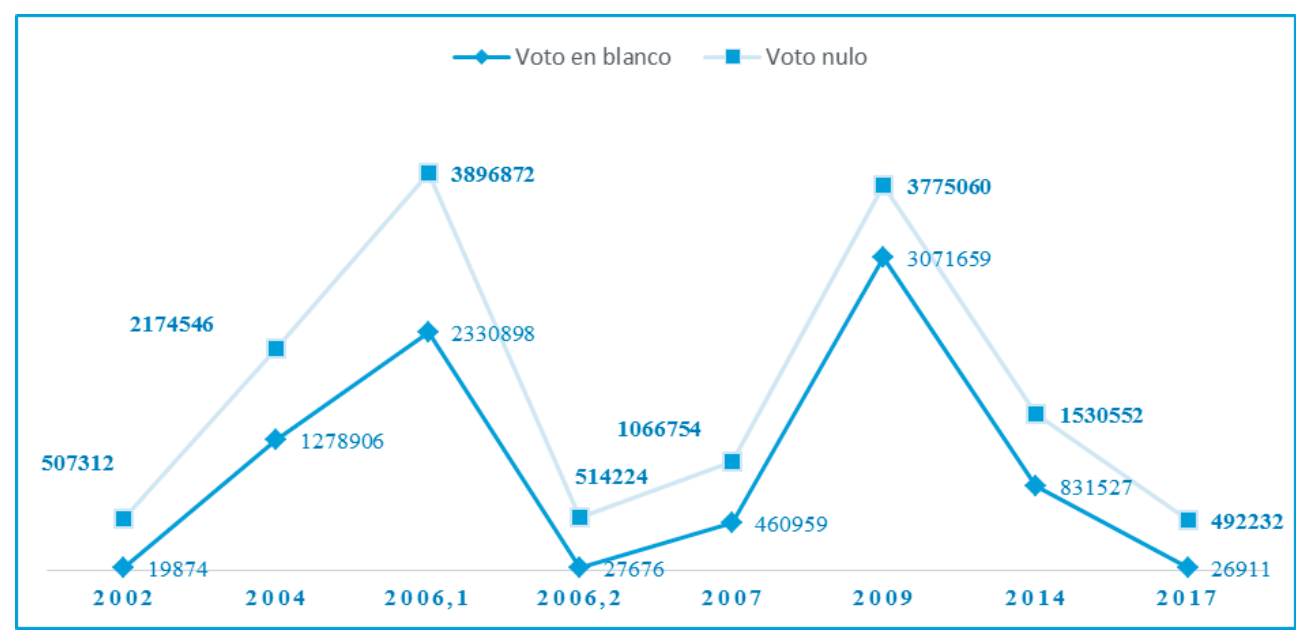

Fuente: Bases de datos de CNE 


\section{LA CANTIDAD DE PARTICIPANTES Y LOS DESAFÍOS PARA LOS ESTRATE- GAS POLÍTICOS}

El término adecuado para la cantidad de candidatos se denomina fragmentación y se conoce también como NEP (número efectivo de partidos), de acuerdo a la publicación del Consejo Nacional Electoral (CNE) denominada “Atlas electoral 2009-2014”, la fragmentación es una medida de la división de fuerzas políticas en una unidad territorial determinada (Figura 3)

"Este indicador revela en que magnitud los votos se dispersan entre las opciones políticas existentes en un territorio, es decir corresponde al número equivalente de organizaciones políticas (OPs) de igual tamaño (con el mismo porcentaje de votos) participantes en una determinada elección". (CNE, 2014, p.350)

En Ecuador la fragmentación a nivel nacional en 2013 creció en un $16.67 \%$ con respecto al grado de fragmentación del 2009.

Figura 3. Fragmentación en Ecuador 2009-2013.

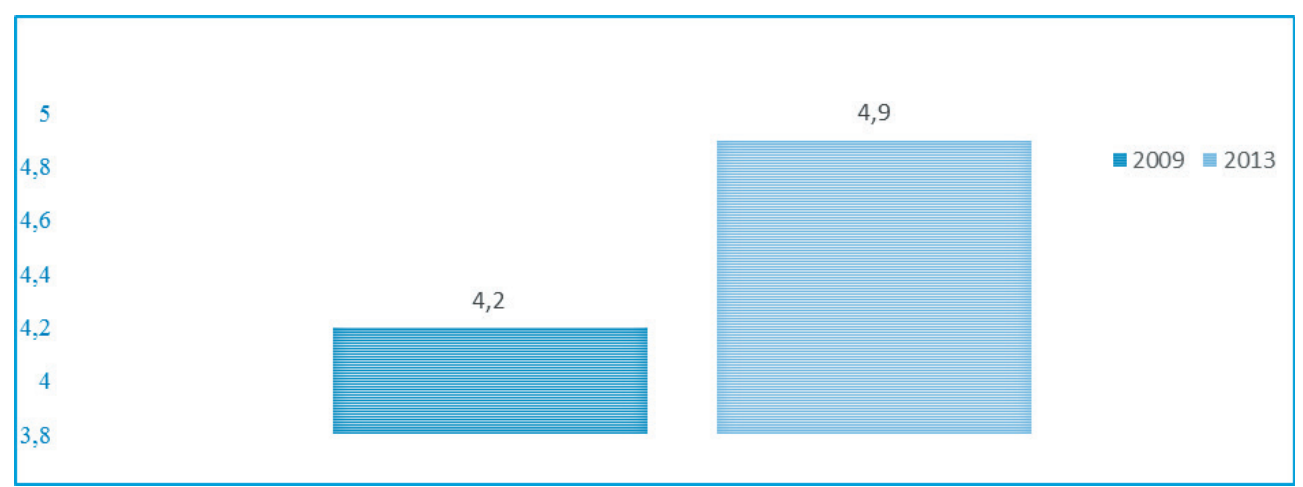

Fuente: CNE (2014)

La llegada de las herramientas digitales, en especial las redes sociales, ha implicado una necesidad de adaptación de los partidos tradicionales, al tiempo que ha permitido un uso diferenciado en los nuevos partidos (López-López; Oñate; Chavero, 2018). Los tiempos modernos dinamizan la Comunicación Política, Blumler (2013) se refirió en principio a la avalancha de comunicación, la abundante información circula en los principales medios de comunicación, a través de Internet, más los dispositivos: en casa, en el trabajo, en la carretera y en la mano.

La competencia intensificada para atraer la atención de la audiencia en tiempos electorales no solo involucra a los contrincantes, en el ciberespacio también están otras temáticas como: deporte, música, moda, cocina, telenovela y otros dramas populares, reality shows, payasadas de celebridades, etc. (Blumler, 2015). En esta situación, los comunicadores políti- 
cos pueden estar bajo presión para pensar más sobre la presentación de sus mensajes y cómo transmitirlos de manera sustancial. "Pero la abundancia también puede estar cambiando la composición de la audiencia para la política, permitiendo que más personas elijan lo que quieren consumir. Aunque, en la ecología de hoy saturada de noticias, incluso a las personas políticamente indiferentes les puede resultar difícil evitar algunos de los bombardeos de material políticamente relevante”. (Blummer, 2013, párr.12).

\section{LAS REDES SOCIALES}

Los primeros antecedentes de la comunicación política en redes sociales en el contexto ecuatoriano aluden a Correa en las elecciones presidenciales de 2006, quien con ayuda de internet (mailing, páginas web,etc.), ocasionó una ruptura en la comunicación política tradicional.

"En 2010 Rafael Correa se unió a Twitter, donde ha desarrollado la mayor actividad en lo que se refiere a redes sociales. En la actualidad, a pesar de haber dejado la Presidencia de Ecuador, sigue haciendo uso de esta red social y gozando de amplia aceptación e interacción con sus seguidores". (Silva; Rodríguez, 2018, p. 530).

En actualidad, los ciudadanos tienen como fuente de información política a las redes sociales Twitter o Facebook, también perciben información a través de servicios como WhatsApp, Instagram o plataformas como YouTube; que desempeñan la función de canal que transmite contenido, "sus esferas de actuación a través de herramientas logarítmicas como la filtración de mensajes, la eliminación de cuentas o la prelación de publicaciones (Enguix-Oliver, 2017) permite concluir que en efecto interfiere en la conformación de las estrategias de comunicación política”. (López-López; Vásquez-González, 2018, p. 1206).

Las redes sociales como Twitter o Facebook se han convertido en una herramienta indispensable en la estrategia de comunicación de los actores políticos durante las campañas electorales (García-Ortega; Zugasti-Azagralos, 2018). El potencial de estas plataformas es el modelo horizontal de comunicación, es decir, la interacción entre ciudadanos y candidatos; sin embargo, el uso de estas plataformas durante las campañas electorales, según García-Ortega y Zugasti-Azagralos (2018) no constituyen el eje central de estrategia de comunicación por tres razones: "la primera los candidatos no interaccionan con los ciudadanos, sino que prima el carácter autorreferencial; la segunda es el uso estratégico de la hibridación entre nuevos medios y medios convencionales; y, la tercera no se perciben dos modelos de gestión de las plataformas claramente diferenciados entre viejos y nuevos partidos". (p.1215).

\section{LA TASA DE CONVERSIÓN COMO MEDIDA DE EFICIENCIA}

La tasa de conversión puede entenderse como el cambio de la visita que realiza el usuario, a la compra que un cliente hace de un determinado producto o servicio (Machaca, 2018), en términos simples, la tasa de conversión mide cuántos de los usuarios que navegan en internet pasaron a ser clientes, como resultado de los elementos de persuasión presentes en las distintas plataformas digitales.

La tasa de conversión puede calcularse dividiendo: número de conversiones totales entre número de visitas totales (Machaca, 2018, p.36); en el caso del presente artículo se 
consideró que las conversiones totales, son iguales a los votos válidos obtenidos, y el número de visitas equivalen al número de seguidores de un determinado candidato.

\section{MATERIALES Y MÉTODOS}

La investigación se elaboró bajo el enfoque cuantitativo, de modalidad no experimental, descriptiva y correlacional, basada en la observación de los reportes de los resultados de las elecciones de alcaldes 2019 publicados en el CNE. Dado que el propósito del presente trabajo fue determinar la relación entre los votos válidos, el ausentismo, los votos nulos, votos blancos y el número de candidatos a las alcaldías del Ecuador. La hipótesis nula sugiere que no existe relación entre los votos válidos, el ausentismo, los votos nulos, votos blancos y el número de candidatos a las alcaldías.

Sin embargo, el presente trabajo nos lleva a plantearnos varias interrogantes sobre, tales como: ¿dónde ganó la indiferencia o la abstención?, ¿la votación de los candidatos tiene alguna relación con el número de candidatos y la abstención?, y ¿cuál fue el impacto de la campaña en las plataformas de Facebook y Twitter sobre el número de votos válidos?

Para validar la hipótesis y al mismo tiempo contestar dichas interrogantes se dividió a la investigación en dos momentos; en el primer momento, la investigación constó de dos etapas, la primera fue la descarga de los 221 reportes de los resultados de la página del CNE, y la segunda, fue la consolidación de los reportes en una sola base de datos de Spss, software que facilitó el cálculo del coeficiente de correlación Rho de Sperman - que se utilizó debido a que las variables son discretas - y la presentación de los principales hallazgos.

El segundo momento de la investigación, tuvo como detonante los desafíos de la cuarta era de la comunicación política que describe Blumler (2013), y también se ejecutó en dos fases, en la primera se busca determinar la existencia de alguna relación entre el número de seguidores de las plataformas Facebook y Twitter y los votos válidos de las elecciones de 2019, en el caso particular de Guayaquil y Quito de los dos candidatos más votados de esas ciudades. Y la segunda fase se analizó el nivel de eficiencia de la estrategia de los candidatos ganadores de Quito y Guayaquil, mediante la tasa de conversión, que se obtuvo de dividir el número de votos válidos (conversiones totales) entre el número de seguidores de las plataformas Facebook y Twitter.

\section{RESULTADOS Y DISCUSIÓN}

\section{Aspectos generales}

El porcentaje promedio de votos en blanco fue de 4.47\%, los votos nulos alcanzaron una media de $6.13 \%$, mientras que la abstención (o ausentismo) alcanzó una media del $15.45 \%$. Solo el 7.24\% de los 221 candidatos a alcalde ganadores obtuvieron una votación válida que es mayor al $50 \%$. 
Tabla 2. Partidos y Movimientos según el número de alcaldías conseguida.

\begin{tabular}{|c|c|c|c|}
\hline \multirow{2}{*}{ Partido Político } & \multicolumn{2}{|c|}{ Número de alcaldías } & \multirow{2}{*}{ Total } \\
\hline & Solo & Alianza & \\
\hline PSC 6 & 9 & 33 & 42 \\
\hline CREO 21 & 9 & 25 & 34 \\
\hline Democracia Sí 20 & 8 & 21 & 29 \\
\hline Alianza PAIS 35 & 10 & 18 & 28 \\
\hline $\begin{array}{l}\text { Movimientos provinciales o } \\
\text { cantonales }\end{array}$ & 23 & & 23 \\
\hline Juntos Podemos 33 & 3 & 17 & 20 \\
\hline Pachakutik 18 & 15 & 4 & 19 \\
\hline SUMA 23 & 8 & 7 & 15 \\
\hline Partido Socialista 17 & 1 & 14 & 15 \\
\hline Centro Democrático 1 & 1 & 13 & 14 \\
\hline Ecuatoriano Unido 4 & 1 & 13 & 14 \\
\hline Unidad Popular 2 & 2 & 12 & 14 \\
\hline Unión Ecuatoriana 19 & 3 & 7 & 10 \\
\hline Fuerza Ecuador 10 & & 9 & 9 \\
\hline Sociedad Patriótica 3 & 3 & 6 & 9 \\
\hline Avanza 8 & 5 & 3 & 8 \\
\hline Izquierda Democrática 12 & 3 & 3 & 6 \\
\hline Justicia Social 11 & 1 & 2 & 3 \\
\hline $\operatorname{mar}-70$ & 1 & 2 & 3 \\
\hline Concertación 51 & 1 & 1 & 2 \\
\hline
\end{tabular}

\section{Fuente: Resultados CNE/ El Universo}

El promedio general de los votos válidos de los nuevos alcaldes fue del 35.18\%, de los 221 candidatos ganadores, solo el $8.14 \%$ son mujeres y de este porcentaje solo el $11.11 \%$ obtuvo una votación igual o mayor al $50 \%$.

El ausentismo ganó en seis cantones con un promedio del 36.76\% (ver figura 4), el dato curioso es la coincidencia con la cantidad de candidatos, el cantón con el mayor número de alcaldables fue La Troncal con 15 participantes. En Biblian por ejemplo, la diferencia entre el ausentismo y el candidato ganador alcanzó 16,18 puntos porcentuales, el promedio 
de la diferencia entre la abstención y los votos válidos de los alcaldes electos es de $9.52 \%$. Figura 4. El ausentismo ganador.

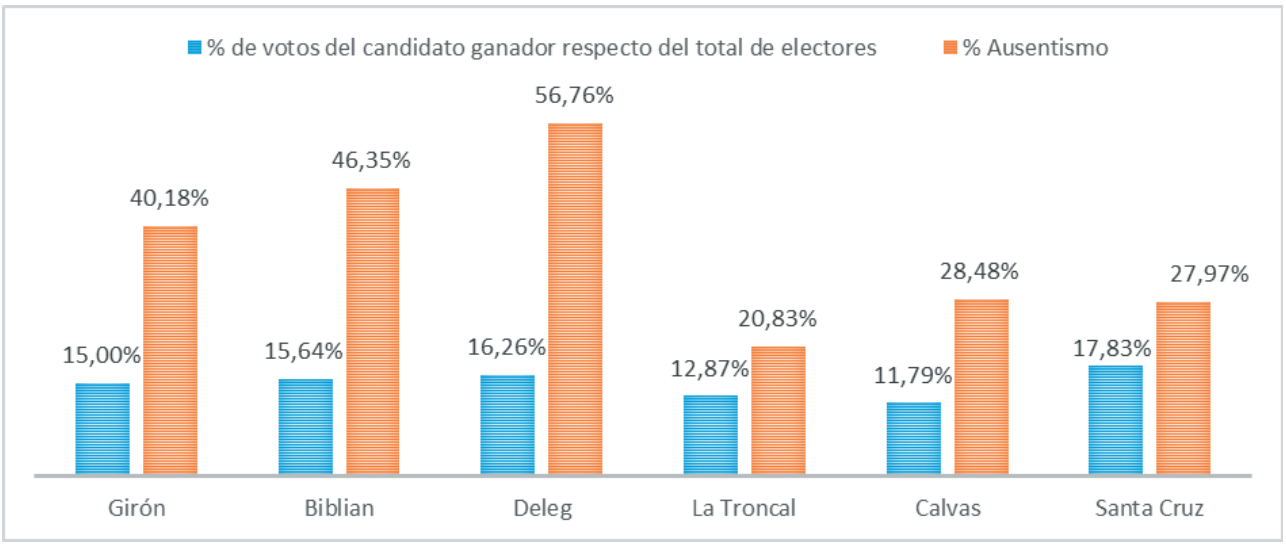

Fuente: CNE (2014).

El comportamiento del voto válido, el voto en blanco, el voto nulo y el ausentismo, es muy irregular en los 221 cantones del Ecuador (ver figura 5); los contextos políticos son únicos para cada cantón. Sin embargo, la tendencia del ausentismo sugiere que, entre los desafíos para los alcaldes electos, esta gobernar con bajos niveles de aprobación por parte de la ciudadanía. Figura 5. Las decisiones de los electores a nivel nacional.

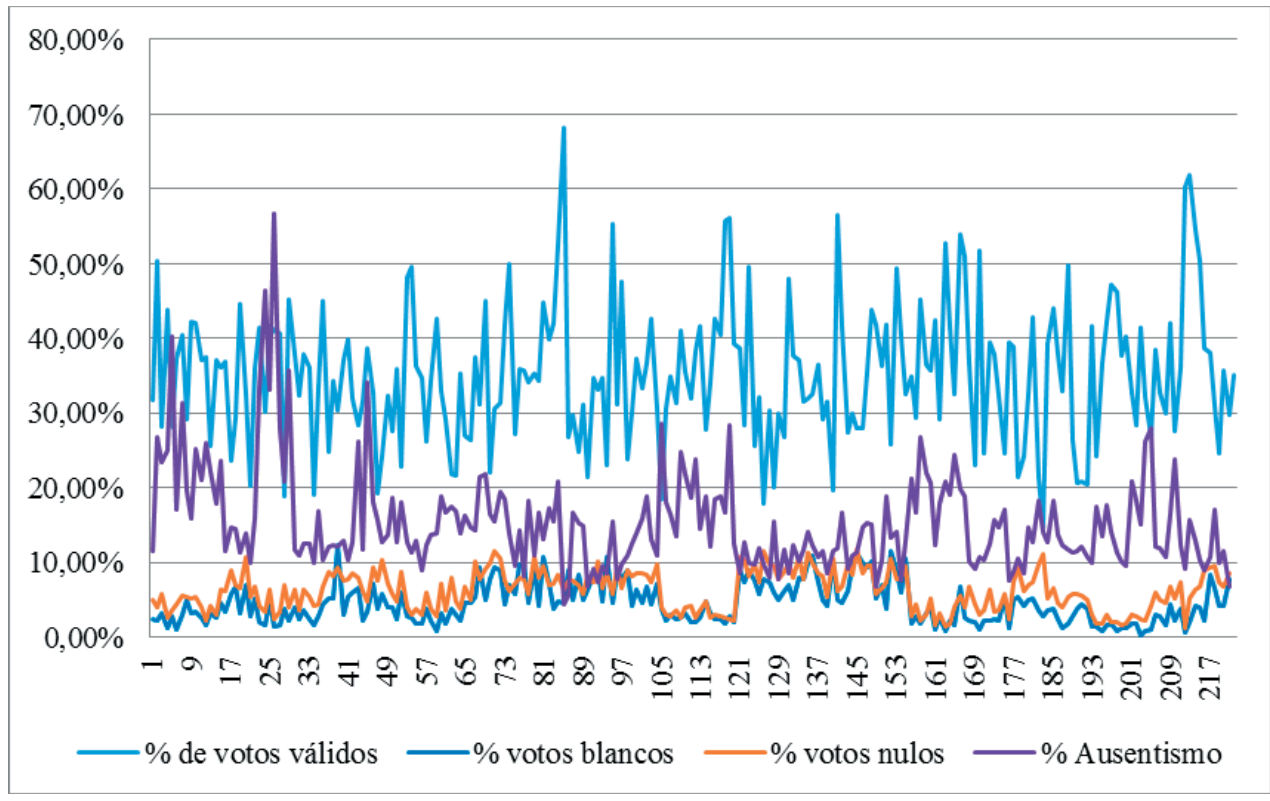

Fuente: Resultados 2019 
En la tabla 3, se muestra el grado de relación entre los votos válidos, votos blancos, votos nulos, el ausentismo y el número de candidatos, por lo tanto debe descartarse la hipótesis nula, puesto que la variable número de candidatos tiene coeficientes de correlación muy significativos con el resto de variables, especialmente con votos blancos (0.771) y votos nulos (0.777). Los votos blancos y nulos también presentan una relación positiva y significativa con el ausentismo (0.850 y 0.867 , respectivamente).

Tabla 3. Matriz de correlación: Rho de Spearman

\begin{tabular}{|l|l|c|c|c|c|c|}
\hline \multicolumn{7}{|c|}{ Matriz de Correlaciones } \\
\hline \multicolumn{1}{|c|}{} & $\begin{array}{c}\text { Votos } \\
\text { Válidos }\end{array}$ & $\begin{array}{c}\text { Votos } \\
\text { Blancos }\end{array}$ & $\begin{array}{c}\text { Votos } \\
\text { Nulos }\end{array}$ & Ausentismo & $\begin{array}{c}\text { Número de } \\
\text { candidatos }\end{array}$ \\
\hline $\begin{array}{l}\text { Rho } \\
\text { de } \\
\text { Spear- } \\
\text { man }\end{array}$ & $\begin{array}{l}\text { Votos } \\
\text { Válidos }\end{array}$ & 1,000 &, $893^{* *}$ &, $914^{* *}$ &, $872^{* *}$ &, $602^{* *}$ \\
\cline { 2 - 8 } & $\begin{array}{l}\text { Votos } \\
\text { Blancos }\end{array}$ &, $893^{* *}$ & 1,000 &, $978^{* *}$ &, $850^{* *}$ &, $771^{* *}$ \\
\cline { 2 - 8 } & $\begin{array}{l}\text { Votos } \\
\text { Nulos }\end{array}$ &, $914^{* *}$ &, $978^{* *}$ & 1,000 &, $867^{* *}$ &, $777^{* *}$ \\
\cline { 2 - 7 } & Ausentismo &, $872^{* *}$ &, $850^{* *}$ &, $867^{* *}$ & 1,000 &, $585^{* *}$ \\
& $\begin{array}{l}\text { Número de } \\
\text { candidatos }\end{array}$ &, $602^{* *}$ &, $771^{* *}$ &, $777^{* *}$ &, $585^{* *}$ & 1,000 \\
\cline { 2 - 7 } & & & & & \\
\hline$* *$ La correlación es significativa al nivel 0,01 (bilateral). & & \\
\hline
\end{tabular}

\section{LOS CANDIDATOS MÁS VOTADOS Y LA COMUNICACIÓN POLÍTICA}

En un intento por explicar las posibles relaciones entre la estrategia desarrollada en redes sociales (Facebook y Twitter) y los resultados de las elecciones 2019 (votos válidos que obtuvieron los dos candidatos más votados), se calculó el coeficiente de Rho de Spearman.

El promedio de seguidores en Twitter y los votos válidos presentan un coeficiente de correlación débil $(0,400)$ (ver tabla 4$)$. La relación más significativa $(0,800)$ ocurre entre el promedio de seguidores en Facebook y votos válidos. Estos hallazgos confirman que, en parte, la estrategia de comunicación política ejecutada en las redes sociales, puede estar relacionada con los resultados en la designación de autoridades por votación popular; además es necesario considerar que la mayoría de los votantes no tiene una cuenta de Twitter. Según la plataforma en el mundo existen 126 millones de usuarios activos, esto probablemente influya en el valor del coeficiente. 
Tabla 4. Matriz de correlaciones

\begin{tabular}{|c|c|c|c|c|c|}
\hline \multicolumn{6}{|c|}{ Matriz Correlaciones } \\
\hline & & & $\begin{array}{c}\text { Votos } \\
\text { válidos }\end{array}$ & $\begin{array}{c}\text { Promedio de } \\
\text { seguidores en } \\
\text { Facebook }\end{array}$ & $\begin{array}{c}\text { Promedio de } \\
\text { seguidores en } \\
\text { Twitter }\end{array}$ \\
\hline \multirow{9}{*}{$\begin{array}{l}\text { Rho } \\
\text { de } \\
\text { Spear- } \\
\text { man }\end{array}$} & \multirow{3}{*}{$\begin{array}{l}\text { Votos } \\
\text { válidos }\end{array}$} & $\begin{array}{l}\text { Coefici- } \\
\text { ente de } \\
\text { correl- } \\
\text { ación }\end{array}$ & 1,000 &, 800 & ,400 \\
\hline & & $\begin{array}{l}\text { Sig. (bi- } \\
\text { lateral) }\end{array}$ & . & 200 & 600 \\
\hline & & $\mathrm{N}$ & 4 & 4 & 4 \\
\hline & \multirow{3}{*}{$\begin{array}{l}\text { Prome- } \\
\text { dio de } \\
\text { segui- } \\
\text { dores en } \\
\text { Face- } \\
\text { book }\end{array}$} & $\begin{array}{l}\text { Coefici- } \\
\text { ente de } \\
\text { correl- } \\
\text { ación }\end{array}$ & ,800 & 1,000 & ,200 \\
\hline & & $\begin{array}{l}\text { Sig. (bi- } \\
\text { lateral) }\end{array}$ & ,200 & . & 800 \\
\hline & & $\mathrm{N}$ & 4 & 4 & 4 \\
\hline & \multirow{3}{*}{$\begin{array}{l}\text { Prome- } \\
\text { dio de } \\
\text { segui- } \\
\text { dores en } \\
\text { Twitter }\end{array}$} & $\begin{array}{l}\text { Coefici- } \\
\text { ente de } \\
\text { correl- } \\
\text { ación }\end{array}$ & ,400 & ,200 & 1,000 \\
\hline & & $\begin{array}{l}\text { Sig. (bi- } \\
\text { lateral) }\end{array}$ & 600 &, 800 & . \\
\hline & & $\mathrm{N}$ & 4 & 4 & 4 \\
\hline
\end{tabular}

En este punto, es importante aclarar que la estrategia de comunicación política es multifactorial, las tasas de conversión para Facebook y Twitter, muestran una parte del basto conjunto de factores que determinan la eficiencia de la comunicación política. Por lo tanto, es un campo de estudio que necesita profundidad en el diseño metodológico, que permita entender cómo los resultados (KPIs) aportaron a las tácticas de campaña utilizadas en las elecciones seccionales de 2019. (Rubiano-Moreno; Barreto-Galeano, 2015).

La tasa de conversión se construyó a partir de dividir los votos válidos entre la media de seguidores de los alcaldables tanto para Facebook como para Twitter. En Facebook (ver tabla 5), por ejemplo, la tasa de conversión más baja es de Jimmy Jairala (47.96\%); mientras que los demás alcaldables obtuvieron un desempeño importante, quizá no influyente. 
Tabla 5. Índice de conversión para Facebook

\begin{tabular}{|l|c|c|c|}
\hline Candidatos & $\begin{array}{l}\text { Promedio de segui- } \\
\text { dores en Facebook* }\end{array}$ & Votos válidos & Tasa de conversión \\
\hline $\begin{array}{l}\text { Cynthia Viteri, Alcaldesa } \\
\text { de Guayaquil electa. }\end{array}$ & 324898 & 714369 & 2,19874853 \\
\hline Jimmy Jairala & 900259 & 431853 & 0,47969862 \\
\hline $\begin{array}{l}\text { Jorge Yunda (Panas de } \\
\text { Yunda), Alcalde de Quito } \\
\text { electo. }\end{array}$ & 61361,2 & 296096 & 4,825459737 \\
\hline $\begin{array}{l}\text { Luisa Maldonado } \\
* \text { El promedio se calculó } \\
\text { en base a los datos } \\
\text { obtenidos de Social } \\
\text { Bakers }\end{array}$ & 19846,5 & 255007 & 12,84896581 \\
\hline
\end{tabular}

En Twitter (ver tabla 6) Jorge Yunda presentó una tasa de conversión casi perfecto (99.55\%), aunque el mejor desempeño corresponde a la ex candidata a Alcaldesa de Quito Luisa Maldonado (1703.11\%).

Tabla 6. Índice de conversión para Twitter.

\begin{tabular}{|l|c|c|c|}
\hline \multicolumn{1}{|c|}{ Candidatos } & $\begin{array}{c}\text { Promedio de } \\
\text { seguidores } \\
\text { en Twitter* }\end{array}$ & Votos válidos & Tasa de conversión \\
\hline $\begin{array}{l}\text { Cynthia Viteri, Alcaldesa de } \\
\text { Guayaquil electa. }\end{array}$ & 262487 & 714369 & 2,721540495 \\
\hline Jimmy Jairala & 182364,4 & 431853 & 2,368077322 \\
\hline $\begin{array}{l}\text { Jorge Yunda, Alcalde de } \\
\text { Quito electo }\end{array}$ & 297433,6 & 296096 & 0,995502862 \\
\hline Luisa Maldonado & 14973 & 255007 & 17,03112269 \\
\hline * El promedio se calculó en base a los datos obtenidos de Social Bakers \\
\hline
\end{tabular}

Por otra parte, los resultados de Luisa Maldonado en la tasa de conversión, puede deberse al apalancamiento que propiciaron las cuentas de Rafael Correa y el movimiento Revolución Ciudadana. Sin embargo, no se debe descartar el uso de boots y trolls, pues constituye una advertencia, que realiza Castañeda (2018, p.193) cuando afirma que "algunas de las prácticas que despliegan los equipos de comunicación de los partidos tergiversan su carácter democrático a través del uso de bots, trolls y otras cuentas falsas dedicadas a manipular los mensajes y controlar sus efectos en la opinión pública”. 


\section{CONCLUSIONES}

A partir de los objetivos del presente artículo, se puede concluir que existe una correlación alta y positiva entre los votos válidos, votos en blanco, votos nulos y el ausentismo, mientras que el número de candidatos presenta coeficientes de 0,$602 ; 0,771 ; 0,777$ y $0,5850,602$; 0,779 y 0,585 con respecto a las variables anteriores; es decir, que dentro de los distintos factores que pueden determinar una votación específica, el número de candidatos es una variable moderadora; ahora bien estos resultados no significan que la relación es estática, al contrario los escenarios en los cuales se desenvuelven los actores del sistema electoral descarta toda certeza y posibilidad de generalizar y predecir resultados, al menos desde la óptica de la presente investigación.

Los índices de conversión de la estrategia de comunicación en Facebook y Twitter sugieren dos interpretaciones: la primera que las redes sociales, en parte, pueden traducir los esfuerzos de la estrategia en votos; y la segunda, que estas plataformas no son relevantes para los estrategas políticos que, en el contexto ecuatoriano, optan por las tácticas de comunicación tradicionales, debido a que los medios, a pesar del surgimiento de las redes sociales, no han podido ser desplazados y gozan de alta credibilidad en los procesos electorales.

Las futuras investigaciones deben procurar el diseño de nuevas metodologías que permitan comprender mejor la influencia de las redes sociales en la designación de autoridades por voto popular, por cuanto existe una tendencia a incrementarse el uso de la comunicación 2.0 en la comunicación política electoral.

\section{REFERENCIAS BIBLIOGRÁFICAS}

AVENDAÑO, O. y SANDOVAL, P. (2016). Political Disaffection and Stability of Electoral Results in Chilean, 1993-2009. Perfiles latinoamericanos, 24(47), 175-198. https:// dx.doi.org/10.18504/pl2447-010-2016

ÁVILA-EGGLETON, M. y GUTIÉRREZ-SÁNCHEZ, H. (2017). "El papel del espacio en la explicación del abstencionismo. Espiral (Guadalajara)", v. 24, n. 69 , pp. 115-154. http://www.scielo.org.mx/scielo.php?script=sci_arttex$t \&$ pid $=$ S1665-05652017000200115\&lng=es\&tlng=es.

BARREDA, M. y RUIZ RODRÍGUEZ, L. M. (2019). Party trust and electoral stability in Latin America. Revista mexicana de sociología, 81(2), 247-273. Recuperado en 23 de diciembre de 2019, de http://www.scielo.org.mx/scielo.php?script=sci_arttext\&pid=S0188-25032019000200247\&lng=es\&tlng=en.

BLUMLER, J. (2013). "The fourth age of political communication”. Keynote address presented at the Workshop on Political Communication Online. Berlin: Free University.

BLUMLER, J. (2015). "Core Theories of Political Communication: Foundational and Freshly Minted". Communication Theory, v. 25, n. 4, pp. 426-438. https://doi.org/10.1111/ comt. 12077 
CASTAÑEDA, V. G. (2018). “Bots, Trolls, Fake News y Memes: Contextos contemporáneos de participación política de los Millennials". https://www.ceenl.mx/educacion/documentos/CEP19/TS1_Certamen\%20de\%20Ensayo\%20Pol\%C3\%ADtico\%20XIX. $p d f$

CAZORLA, A.; RIVERA, J. M. y JARÁIZ, E. (2017). "La abstención electoral en las elecciones al Parlamento Europeo de 2014: análisis estructural de sus componentes". Revista Española de Investigaciones Sociológicas, n. 159, pp. 31-50. http://dx.doi. org/10.5477/cis/reis.159.31

CLARKE, H.; SANDERS, D.; STEWART, M. y WHITELEY, P. (2011). "Valence Politics and Electoral Choice in Britain, 2010". Journal of Elections, Public Opinion \& Parties, v. 21, n. 2, pp. 237-253. https://doi.org/10.1080/17457289.2011.562614

CNE (2014). “Atlas electoral 2009-2014”. Quito: Consejo Nacional Electoral. http://cne. gob.ec/es/estadisticas/publicaciones/category/136-atlas-electoral-2009-2014

COUTIGNO, C. A. (2018). "Cultura política ciudadana y abstención electoral en el municipio fronterizo de Tijuana". Estudios Fronterizos, v. 19. https://doi.org/10.21670/ ref. 1807007

DOMPER-LASÚS, C. (2018). "El franquismo a través de las urnas. Metodología, fuentes y retos para una aproximación electoral al régimen de Franco", Política y Sociedad, 55(1), pp. 115-134. https://doi.org/10.5209/POSO.54803

El Universo (1 de abril, 2019). “Alianzas enmascaran la votación por partidos en las últimas elecciones seccionales en Ecuador". https://www.eluniverso.com/noticias/2019/04/01/nota/7262930/alianzas-enmascaran-votacion-partidos

GARCÍA-ORTEGA, C. y ZUGASTI-AZAGRA, R. (2018). "Gestión de la campaña de las elecciones generales de 2016 en las cuentas de Twitter de los candidatos: entre la autorreferencialidad y la hibridación mediática". El profesional de la información, v. 27, n. 6, pp. 1215-1224. https://doi.org/10.3145/epi.2018.nov.05

GIULIANI, M. (2019). "Making sense of pollsters' errors. An analysis of the 2014 second-order European election predictions", Journal of Elections, Public Opinion and Parties, v. 29, n. 2, pp. 162-178. https://doi.org/10.1080/17457289.2018.1466786

LÓPEZ-LÓPEZ, P. C.; OÑATE, P. y CHAVERO, P. (2018). “El horse-race campaign frente al issue-campaign en la agenda digital: elecciones presidenciales en América Latina en el periodo 2015-2017”. Revista Ibérica de Sistemas e Tecnologias de Informação, v. 16, n. 11, pp. 270-282. http://www.risti.xyz/issues/ristiel6.pdf

LÓPEZ-LÓPEZ, P. C y VÁSQUEZ-GONZÁLEZ, J. (2018). “Agenda temática y Twitter: elecciones presidenciales en América Latina durante el período 2015-2017”. El 
profesional de la información, v. 27, n. 6, pp. 1204-1214. https://doi.org/10.3145/ epi.2018.nov.04

LOZANO-GARCÍA, M. A. y RODRÍGUEZ-CASALLAS, D. (2015). "Voto en blanco, voto nulo y abstención: expresión de rechazo de la ciudadanía boyacense, en los comicios parlamentarios del 2014". Revista VIA IURIS, v.18, pp. 135-150. http://www.redalyc.org/pdf/2739/273944646007.pdf

MACHACA, H. (2018). Impacto de las estrategias de marketing digital en redes sociales de empresas distribuidoras de vehículos motorizados en el consumidor de la ciudad de Juliaca 2017 (Tesis de grado). Universidad Peruana Unión. Juliaca-Perú. Recuperado de: https://repositorio.upeu.edu.pe/bitstream/handle/UPEU/1513/Hilario_Tesis_Licenciatura_2018.pdf?sequence $=5 \&$ isAllowed $=\mathrm{y}$

MILANESE, J. P.; ABADÍA, A. y MANFREDI, L. (2016). "Elecciones regionales en Colombia 2015: Análisis de las nuevas Gobernaciones y Asambleas Departamentales". Revista Uruguaya de Ciencia Política, v. 25, n. 1, pp. 63-89. http://www.redalyc. org/articulo.oa? $\mathrm{id}=297346770001$

MÜLLER, S. y JANKOWSKI, M. (2018). "Do voters really prefer more choice? Determinants of support for personalised electoral systems". Journal of Elections, Public Opinion and Parties, v. 29, n. 2, pp. 262-281. https://doi.org/10.1080/17457289.2 018.1515211

OCHOA-VILLA, R. (2015). “Comportamiento electoral de los colombianos durante las elecciones presidenciales del año 2010". Jurídicas CUC, v. 11, n. 1, pp. 221-232. https:// revistascientificas.cuc.edu.co/juridicascuc/article/view/JURIDICAS\%20CUC

RUBIANO-MORENO, J. y BARRETO-GALEANO, M. (2015). "Relación entre variables sociodemográficas, psicosociales y de marketing político en la conducta de voto y abstencionismo de jóvenes". Revista Perspectiva Empresarial, v. 2, n. 1, pp. 61-74. https://doi.org/10.16967/rpe.v2n1a4

SILVA, J. y RODRÍGUEZ, C. (2018). "Presencia y popularidad de los candidatos presidenciales de Ecuador en Facebook y Twitter en las elecciones generales de 2017”. En: La Innovación de la Innovación: Del Medio al Contenido Predictivo. Actas del III Simposio Internacional sobre Gestión de la Comunicación, pp. 526-544. https:// xescom2018.files.wordpress.com/2019/01/actas-2-edicion-completo-526-544.pdf

SOUTHERN, R. y LEE, B. (2018). "Politics as usual? Assessing the extent and content of candidate-level online campaigning at the 2015 UK general election". Journal of Elections, Public Opinion and Parties, v. 29, n. 2, pp. 179-198. https://doi.org/10.1 080/17457289.2018.1515209 
SUlMONT, D. (2018). Is There a Programmatic Vote in Elections in Weak Political Party Systems? An Analysis of the 2016 Peruvian Presidential Election. Revista de ciencia política (Santiago), 38(3), 429-457. https://dx.doi.org/10.4067/S0718090X2018000300429

VASQUEZ-FERIA, A. G. M. E. (2019). “Expectativas políticas de jóvenes abstencionistas: el caso del distrito electoral federal X de Veracruz, México". Revista de Ciencias Sociales, v. 32, n. 44, pp. 151-170. https://dx.doi.org/10.26489/rvs.v32i44.7 